\title{
Trapped atoms in cavity QED: coupling quantized light and matter
}

\author{
R Miller, T E Northup, K M Birnbaum, A Boca, A D Boozer \\ and $\mathrm{H} J$ Kimble \\ Norman Bridge Laboratory of Physics 12-33, California Institute of Technology, Pasadena, \\ CA 91125, USA \\ E-mail: hjkimble@caltech.edu
}

Received 2 November 2004

Published 25 April 2005

Online at stacks.iop.org/JPhysB/38/S551

\begin{abstract}
On the occasion of the hundredth anniversary of Albert Einstein's annus mirabilis, we reflect on the development and current state of research in cavity quantum electrodynamics in the optical domain. Cavity QED is a field which undeniably traces its origins to Einstein's seminal work on the statistical theory of light and the nature of its quantized interaction with matter. In this paper, we emphasize the development of techniques for the confinement of atoms strongly coupled to high-finesse resonators and the experiments which these techniques enable.
\end{abstract}

(Some figures in this article are in colour only in the electronic version)

\section{From Einstein to cavity QED}

In the years prior to his seminal 1905 papers, Albert Einstein had given much thought to the statistical properties of electromagnetic fields [1], especially with regard to the theory of black-body radiation developed by Max Planck [2]. Einstein realized that the quantization of light-particularly the creation and annihilation of 'light quanta'-is something more fundamental than a tacit consequence of the assumption that the total energy of a black-body is discretely distributed between a set of microstates. Beginning in 1905 with On a heuristic point of view about the creation and conversion of light [3] and in four subsequent papers on quantization [4-7], he laid the foundations of the 'old quantum theory' [8], summarized in what is commonly referred to as the 'light quantization hypothesis':

... the energy of a light ray emitted from a point [is] not continuously distributed over an ever increasing space, but consists of a finite number of energy quanta which are localized at points in space, which move without dividing, and which can only be produced and absorbed as complete units [3]. 
He argued that the existence of light quanta was essential to Planck's hypothesis. In his treatment of the problem, he developed a formalism based upon the inversion of Boltzmann's law to describe the statistical variances in the energy of a black body. Using this technique, he was able to separate these energy fluctuations into the sum of two quantities, one representing the fluctuation of a number of particles and the other corresponding to the variances of a classical wave $[6,9]$. The wave-particle duality to which Einstein alluded is, of course, now one of the tenets of modern quantum mechanics. These early papers also began, for the first time, to broach phenomenological problems in terms of quantized light. In a very literal sense, Einstein's descriptions of the photoelectric effect and the ultraviolet photoionization of gases [3] constitute the first applications of quantum electrodynamics as well as the first microscopic (rather than statistical) descriptions of quantized electromagnetic phenomena.

While the same could be said of vast expanses of modern physics, much of the intellectual underpinnings of cavity quantum electrodynamics are certainly due to this early work by Einstein. Einstein wrote

The wave theory of light which operates with continuous functions in space has been excellently justified for the representation of a purely optical phenomena and it is unlikely ever to be replaced by another theory. One should, however, bear in mind that optical observations refer to time averages and not to instantaneous values and notwithstanding the complete experimental verification of the theory of diffraction, reflection, refraction, dispersion and so on, it is quite conceivable that a theory of light involving the use of continuous functions in space will lead to contradictions of experience, if it is applied to the phenomena of the creation and conversion of light [3].

As will be described in the following sections, an atom strongly coupled to the mode of a resonant cavity is precisely one such situation. In fact the atom-cavity system is uniquely well-suited to the efficient generation of single photons, a phenomenon wherein Einstein's 'contradictions of experience' certainly dominate. The quantized description of light is manifestly that which is needed to describe cavity QED.

In what follows, we introduce cavity quantum electrodynamics in the regime of strong coupling. With an emphasis on the current state-of-the-art experiments, particularly those carried out by our group at Caltech, involving atoms trapped within a optical cavity, we also review the evolution of experiments in cavity QED and discuss future research directions.

\section{Fundamentals of the cavity QED system}

\subsection{Introduction}

Cavity quantum electrodynamics explores the measurement and control of quantized electromagnetic fields and atomic systems coherently coupled inside an electromagnetic resonator. A simple, representative model of the cavity QED system, as illustrated in figure 1, consists of exactly one two-state atomic system at rest within the mode of a resonator formed by two spherical mirrors. The dynamics of this system are described by the well-known Jaynes-Cummings Hamiltonian [10,11] which is composed of the sum of separate terms for both the atom and field as well as a third term which arises from the total atom-field dipole interaction:

$$
\hat{H}=\frac{1}{2} \hbar \omega_{A} \hat{\sigma}_{z}+\hbar \omega_{C}\left(\hat{a}^{\dagger} \hat{a}+\frac{1}{2}\right)+\hbar g(\mathbf{r})\left(\hat{a}^{\dagger} \hat{\sigma}+\hat{a} \hat{\sigma}^{\dagger}\right) .
$$

The operators $\left(\hat{\sigma}_{z}, \hat{\sigma}^{\dagger}, \hat{\sigma}\right)$ are the Pauli operators corresponding to inversion, raising and lowering of the atomic state, while $\left(\hat{a}^{\dagger}, \hat{a}\right)$ are the creation and annihilation operators for 


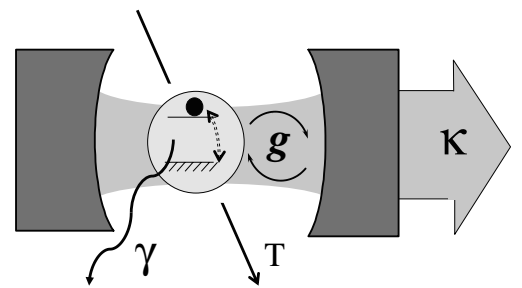

Figure 1. Characteristic parameters of atom, cavity, and environment: $\kappa$, the rate of decay of the cavity field; $\gamma$, the rate at which the atomic dipole radiates into modes other than the cavity field mode; $T$, the transit time of an atom through the cavity mode; and $g$, the rate of coherent atom-field coupling. Strong coupling requires that $g /\left(\gamma, \kappa, \frac{1}{T}\right) \gg 1$.

photons in the coupled mode of the resonator. $\left(\omega_{A}, \omega_{C}\right)$ are the resonant frequencies of the atom and cavity, respectively.

The magnitude of the coupling is a function of the atom's position, $\mathbf{r}$, within the standing wave structure of the mode and is given by

$$
g(\mathbf{r})=\left(\frac{\mu^{2} \omega_{C}}{2 \hbar \epsilon_{0} V_{M}}\right)^{\frac{1}{2}} U(\mathbf{r}) \equiv g_{0} U(\mathbf{r}) .
$$

Here, $\mu$ is the atomic dipole matrix element, $V_{M}$ is the resonant mode volume, a geometric parameter of cavity, and $U(\mathbf{r})$ is the cavity mode function, defined such that $V_{M}=$ $\int|U(\mathbf{r})|^{2} \mathrm{~d}^{3} x$. The coupling coefficient $2 g_{0}$ is known as the single-photon Rabi frequency and represents the maximum rate at which one quantum of excitation is exchanged between atom and field [12].

This idealized model captures the essential dynamics of the interaction but makes no accounting of the dissipative processes which naturally accompany real cavities and atoms. The dominant loss associated with the cavity results from the leakage of photons through the mirrors at a rate $2 \kappa$, where $\kappa$, the frequency half-width of the resonant mode, is specified by the quality factor of the cavity,

$$
Q=\frac{\omega_{C}}{2 \kappa} \text {. }
$$

A second dissipative channel is the result of spontaneous emission from the atom into field modes other than that which is preferentially coupled to the resonator. In general, there are two distinct atomic decay rates along the transverse and longitudinal directions of the cavity $\left(\gamma_{\perp}, \gamma_{\|}\right)$, both of which are functions of the position of the atom, $\mathbf{r}$ [13]. In practice, with current state-of-the-art optical Fabry-Perot cavities this position dependence is largely negligible. In this case, the angle subtended by the mode of the cavity is small, and the transverse decay rate is very closely approximated by the atomic free space decay rate, $\gamma_{\perp}=\gamma=\gamma_{\|} / 2$ (this is not generally true, however, for cavities of different geometries [14-16]). A third consideration is the length of time, $T$, during which the atom resides within the cavity mode, after which no coherent evolution is possible. While each of these three dissipative channels damp the desired quantum evolution of the system, it should be noted that they do not necessarily stand on equal footing. In particular, the rate $\kappa$ is characterized by emission of the cavity field into one, well-defined spatial mode which facilitates efficient readout of the state of the coupled system. The other two rates, $\left(\gamma, T^{-1}\right)$, represent, in general, irreversible loss of information to the environment. As photons are coupled into a continuum of experimentally inaccessible spatial modes, and as atoms leave the cavity volume (though not necessarily so in the microwave domain), it is difficult to extract useful information from the system. 
The description, above, is generally applicable to cavities with resonant frequencies ranging from the microwave [11] to optical regimes and which may be coupled to appropriate two-level systems as diverse as Cooper pair boxes [17, 18] and Rydberg atoms [19, 20]. Whereas high-finesse Fabry-Perot cavities coupled to alkali atoms in the optical regime will be the focus of this discussion, broad reviews of cavity QED in this and other regimes are available $[12,21]$.

\subsection{Strong coupling}

As we have seen, cavity QED is parametrized by four rates $\left(g_{0}, \kappa, \gamma, T^{-1}\right)$. In order to emphasize the coherent evolution of the system, it is useful to require that the coupling coefficient dominate dissipation:

$$
g_{0} /\left(\gamma, \kappa, T^{-1}\right) \gg 1 .
$$

This condition is commonly known as the strong coupling criterion [12].

It is instructive to explore strong coupling in terms of two dimensionless parameters known as the critical photon and atom numbers. The critical (or saturation) photon number describes the number of photons such that for a cavity of a given geometry the intracavity optical intensity is sufficient to saturate the atomic response $\left(I_{\text {sat }}\right)$ :

$$
n_{0}=\frac{\gamma^{2}}{2 g_{0}^{2}}
$$

Similarly, the critical atom number describes the number of strongly coupled atoms necessary to affect appreciably the intracavity field:

$$
N_{0}=\frac{2 \kappa \gamma}{g_{0}^{2}}
$$

Many quantum optical systems - lasers for instance, with $\sqrt{n_{0}} \sim 10^{3}-10^{4}$ - have large critical parameters and therefore adding or removing one photon or atom does not significantly alter the dynamics as a whole. In these systems, the coherent coupling parameter $g_{0}$ is scaled away as processes approach the semi-classical regime. By contrast, the necessary (though not sufficient) criteria for strong coupling are that $\left(n_{0}, N_{0}\right) \ll 1$. This means that in the regime of strong coupling, single quanta dominate the dynamics of the system such that the interaction between atom and photon can be manifestly nonclassical and nonlinear for single atoms and photons. Strong coupling thereby provides a powerful tool for the study of quantum optics as well as the interaction of the quantized electromagnetic field with matter.

\subsection{The Jaynes-Cummings ladder}

In the strong coupling regime, coherent interaction dominates dissipation and so the JaynesCummings Hamiltonian (1) provides a foundation for the description of the evolution of the system. Allowing the cavity mode to be resonant with the atomic transition frequency $\left(\omega_{C}=\omega_{A}\right)$ and diagonalizing (1) yields a set of eigenstates for the system,

$$
| \pm\rangle_{n}=\frac{1}{\sqrt{2}}(|g, n\rangle \pm|e, n-1\rangle),
$$

where $(g, e)$ denote the ground and excited states of the atom and there are $n$ quanta of excitation in the system. These are the dressed states of the Jaynes-Cummings model and represent the equal distribution of excitation between atom and field with corresponding energy eigenvalues $E_{ \pm}=n \hbar \omega \pm \sqrt{n} \hbar g(\mathbf{r})$. 
Experimentally, as an atom enters the mode of the cavity the transmission and absorption spectra in the weak-field limit will no longer exhibit a single, empty-cavity resonance at $\omega=\omega_{C}$ but instead a two-peaked structure with maxima at $\omega=\omega_{C} \pm \sqrt{n} g(\mathbf{r})$, corresponding to the energy eigenvalues of $| \pm\rangle_{1}$ for one quantum of excitation. This characteristic spectral feature is known as the vacuum-Rabi splitting and serves as a hallmark of strong coupling.

\subsection{Cavities in the laboratory}

While defining the criteria for strong coupling is a relatively simple matter $\left(g_{0} \gg\left(\gamma, \kappa, T^{-1}\right)\right)$, realizing cavities which in practice meet these criteria is a decidedly more complicated task. Over the past 25 years, experiments in optical cavity QED have pushed progressively farther into the regime of strong coupling such that typical values for state-of-the-art optical cavities today are $n_{0} \approx 10^{-3}-10^{-4}$ photons and $N_{0} \approx 10^{-2}-10^{-3}$ atoms. These cavities are of FabryPerot geometry and consist of two superpolished spherical mirror substrates which have been coated with a highly reflective stack of dielectric layers $(R=0.9999984$ is a representative value for the reflectivity of one such state-of-the-art mirror) [22]. The reflectivity of these mirrors is sufficiently large that the inter-mirror spacing (and therefore the mode volume, $V_{m}$ ) can be made relatively small without $\kappa$ growing larger than $g_{0} \propto V_{M}^{-1 / 2}$. Current experiments underway in our group at Caltech involve atomic caesium coupled to a cavity of length $L=42.2 \mu \mathrm{m}$ and with mirrors of radius of curvature $R=20 \mathrm{~cm}$ such that

$$
\left(g_{0}, \kappa, \gamma\right)=(34,4.1,2.5) \mathrm{MHz}
$$

well into the regime of strong coupling. The finesse of this cavity at the D2 line in atomic caesium ( $\lambda=852.4 \mathrm{~nm}$, made resonant with the $\mathrm{TEM}_{00}$ mode of the cavity) is $\mathcal{F}=4.2 \times 10^{5}$, and the critical parameters are $n_{0}=0.0029$ and $N_{0}=0.018$. In future experiments, it may be possible to achieve even higher finesse by coupling to the whispering gallery modes of quartz microspheres [14-16] or microtoroidal resonators [23, 24] or to photonic bandgap resonators $[25,26]$.

\section{Experiments in the regime of strong coupling}

In the following sections, we briefly review the experimental evolution of optical cavity QED in the regime of strong coupling, particularly with regard to those techniques developed by our group at Caltech. The goal is to set the stage for a description of the new set of tools made available by the recent marriage of atom-cavity systems and laser cooling and trapping. A common theme throughout the discussion will be the quest to localize and isolate a single atom which is strongly coupled to the cavity mode, as required by, for instance, a variety of schemes for implementing quantum computation and communication protocols $[27-30,25]$.

\subsection{Early work}

The first observations of atoms strongly coupled to optical resonators came in the early 1990s with experiments involving atomic beam transits through the mode of a high-finesse cavity [31, 32]. The average duration of each single atom transit was $T=0.4 \mu \mathrm{s}$ and the flux of the beam was adjustable such that the average intracavity atom number $\bar{N} \sim 1$. By measuring the transmission of a weak probe of variable detuning about the caesium $\left(6 S_{1 / 2}, F=4 \rightarrow 6 P_{3 / 2}, F^{\prime}=5^{\prime}\right)$ transition, the vacuum-Rabi splitting for one atom (on average) was observed for the first time, albeit weighted over a range of values for 

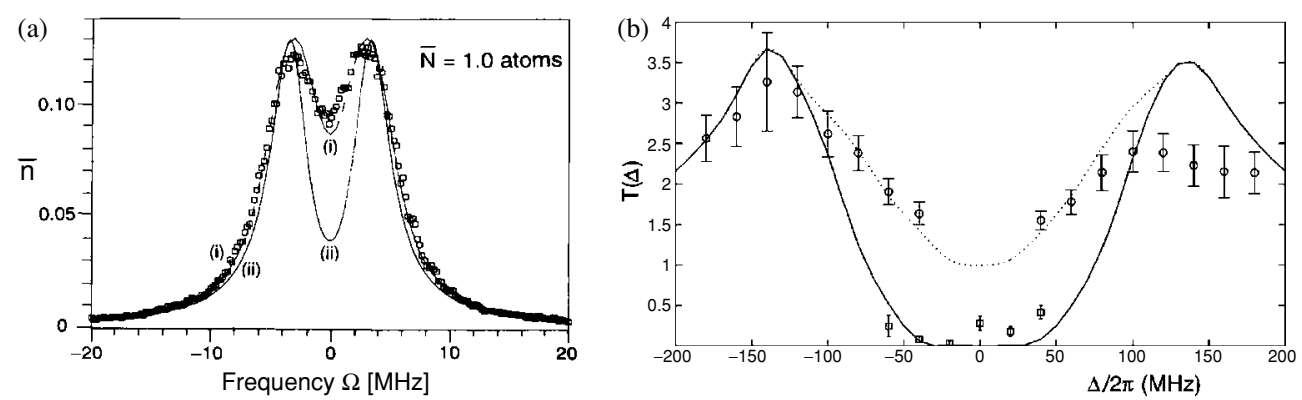

Figure 2. Early one-atom vacuum-Rabi splitting measurements. (a) The vacuum-Rabi splitting for $\bar{N}=1$ as measured with an atomic beam by Thompson et al [32]. Curve (i) represents the theoretical transmission averaged over the relevant range of values for $g(\mathbf{r})$ and $N$, while (ii) describes precisely one atom with maximal coupling $g_{0}$. (b) Vacuum-Rabi spectrum measured using individual atomic transits from a MOT, as described by Hood et al [37]. The solid line is the predicted spectrum based upon evolution of the master equation for maximally coupled atoms while the dashed line represents couplings $g(\mathbf{r})<g_{0}$.

$g(\mathbf{r})$ and an ensemble of atoms (figure 2(a)) [32, 33]. Similar optical normal-mode spectra have been obtained by the group of Feld using a beam of barium atoms [34], while direct observation of atom-field Rabi nutation in the microwave domain was made by the group of Haroche using a beam of rubidium atoms in Rydberg excited states [35].

The brief duration of each transit and uncertainty in the instantaneous rate of atom-field coupling presented a significant limitation on the amount of information made available per atom in these atomic beam experiments [32-35]. Subsequent experiments instead made use of a cloud of cold atoms cooled to sub-Doppler temperatures in a magneto-optic trap (MOT) located a few millimetres above the cavity. When the MOT is released, some fraction of the cold atoms fall between the mirrors, and it is possible to observe in real time their individual trajectories and the durations of their transits [36]. Using this technique, the vacuum-Rabi spectrum (figure 2(b)) was extracted from the transmission of a weak probe interacting with many individual transits (each of duration $T \approx 100 \mu \mathrm{s}$ ) on an atom-by-atom basis [37]. Likewise, this technique has also enabled measurements of the nonlinear optical response of individual atoms to drive fields corresponding to $\bar{n} \ll 1$ intracavity photon [37]. It should be noted that while each individual atom contributes significantly more information to the spectrum in this experiment than in those with atomic beams, the data in figure 2(b) is still the result of an ensemble average over many atoms.

\subsection{The atom-cavity microscope}

By way of the marriage of laser cooled atomic sources and cavity QED, as discussed above, has come an exciting new regime in which the kinetic energy $K$ of atoms in transit through the cavity is comparable to the energy $\hbar g(\mathbf{r})$ associated with the atom-field coupling. In this domain, the presence of just one photon is sufficient to alter profoundly the atom's centre-of-mass motion. Indeed if $\hbar g_{0}>K$ and the cavity is slightly detuned from the bare atomic resonance, then the dressed state $|-\rangle_{1}$, as discussed above, is confined by an attractive pseudopotential due to the intracavity field [38-40]. The pseudopotential wells are of depth determined as a function of the probe intensity and the atom-cavity detuning, and have facilitated atomic trapping times $T \sim 0.5 \mathrm{~ms}$. 

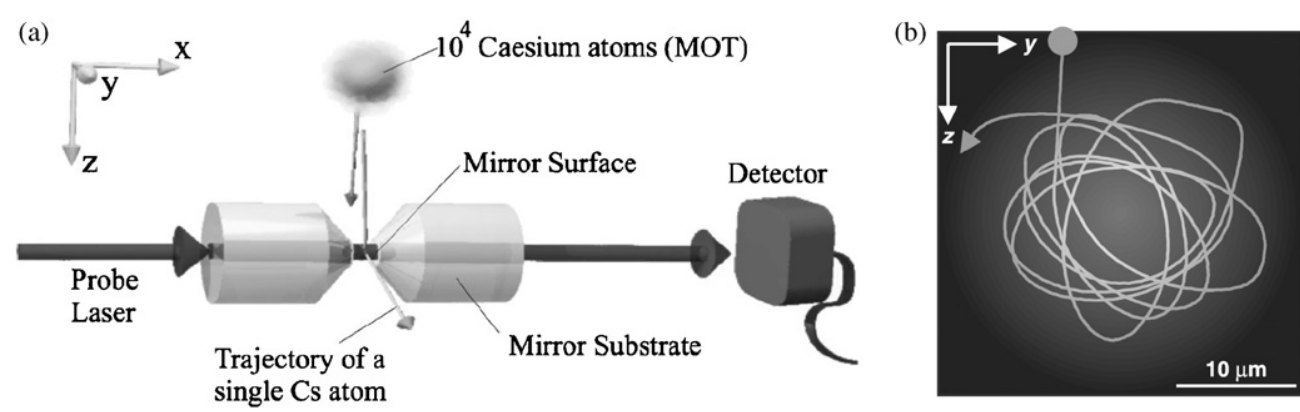

Figure 3. Diagram of the atom-cavity microscope. (a) Cold atoms are delivered to the cavity mode by releasing the contents of a magneto-optic trap (MOT) a few millimetres above the cavity. The transmission of weak probe beam is recorded using balanced heterodyne detection [38]. (b) A reconstruction of the trajectory of a single atom bound to the quantized intracavity field with $\bar{n}=1$ photon. Animations of transits reconstructed using this technique are available at http://www.its.caltech.edu/ qoptics/atomorbits/.

This technique for intracavity confinement enabled the so-called atom-cavity microscope, a protocol for reconstructing the trajectories of single atoms based upon the variations in transmission of a weak probe beam (which also generates the trapping pseudopotential). Experimentally, as a caesium atom enters the mode of the cavity its presence is detected in real time. The resulting signal is used to switch rapidly the intensity of the probe, which is detuned to the red vacuum-Rabi sideband at $\left(\omega_{A}-\hbar g_{0}\right)$, in order to form the trap, pushing atoms towards larger values of $g(\mathbf{r})$. The transmitted probe field is then monitored and recorded via heterodyne detection. For each individual atomic transmission profile, which is indicative of the instantaneous rate of coherent coupling to field, it is then possible to extract information about the orbit in which the confined atoms move (such as that shown in figure 3(b)). This work represents a first step towards addressing the motion of an atom strongly coupled to a cavity, but is limited in the sense that the QED interaction and the trapping potential are intertwined, precluding the ability to address and control the state of the atom while confined by the trap.

\subsection{State-insensitive cooling and trapping}

While atomic confinement using the quantized cavity QED field offers an important advance towards the realization of well-localized, trapped atoms, it remains preferable to decouple the trap from the QED interaction. Towards this goal, a number of groups [41-44] have successfully implemented optical dipole force traps (also known as far off-resonant traps or FORTs) consisting of a far-detuned optical beam able to induce a dissipative, attractive force on an atom and yet only weakly drive atomic transitions.

The principle of operation for a FORT, as applied to a simple two-level atomic model, is rather straightforward [45]. The FORT beam, detuned by $\delta$ from the resonant frequency of the transition, $\omega_{A}$, induces an ac-Stark shift,

$$
\Delta E_{g, e}= \pm \frac{3 \pi c^{2} \gamma}{\omega_{A}^{3}} \frac{I(\mathbf{r})}{\delta},
$$

of equal magnitude but opposite sign for each of the excited and ground states. For a reddetuned field $(\delta<0)$, an atom in its ground state with kinetic energy $K<U_{\text {dip }}=\Delta E_{g}(\mathbf{r})$ is confined in a potential which varies spatially as the intensity of the FORT beam (likewise, an atom in its excited state will, rather undesirably, see a repulsive potential). This process 
is readily extrapolated to more complicated, multilevel systems such as the alkali atoms commonly used in cavity QED, though the overall functional form of (9) remains similar [46].

The earliest implementations of intracavity FORT fields for cavity QED used lasers at frequencies an integer number of cavity free spectral ranges away from the probe field [44]. In so doing, a cavity which is length-stabilized to be resonant with the $\mathrm{TEM}_{00}$ mode of the probe will simultaneously support the $\mathrm{TEM}_{00}$ mode of the FORT as well. The resultant intracavity standing wave provides a series of potential wells into which sufficiently cold atoms can be loaded. The first experiment of this sort involved trapping caesium in a FORT with $\lambda_{\text {FORT }}=869 \mathrm{~nm}$, characterized by a trap lifetime $T=28 \mathrm{~ms}$, which was evidently limited by parametric heating and FM-to-AM noise conversion due to the relatively high finesse of the cavity at the FORT wavelength.

These early difficulties were remedied by choosing a value $\lambda_{\text {FORT }}$ for which the differential ac-Stark shift between excited and ground states was very nearly zero and at which the cavity mirrors were less reflective. Certain atomic lines, including the caesium D2 transition, have the property that for a narrow range of wavelengths the ac-Stark shifts of the excited and ground state manifolds are in the same (trapping) direction [47-49]. By considering couplings to the full manifold of caesium excited states it is possible to recognize a nearly state-insensitive trapping potential (i.e., with only small differential ac-Stark shifts) at $\lambda_{\text {FORT }}=935.6 \mathrm{~nm}$, colloquially referred to as the 'magic' wavelength of caesium [50, 41]. Indeed, it has been shown that an intracavity FORT at this wavelength with a depth corresponding to $U_{0} \approx 3 \mathrm{mK}$ allows for trap lifetimes $T \approx 3 \mathrm{~s}$ 'in the dark' (i.e., in the absence of QED fields), likely limited by the rate of collision with background gas, and $T \approx 1 \mathrm{~s}$ even in the presence of a probe field [41]. This technique constitutes a milestone in optical cavity QED, opening the door to a myriad of experiments involving one-and-the-same atom. These exciting new experiments are to be discussed in the next section.

The use of optical dipole traps for localization of atoms within the mode of a high-finesse cavity has developed rapidly in the past two years. In addition to the work described above, the group of Rempe at the Max Planck Institute in Garching has also implemented the co-resonant FORT technique at $\lambda_{\text {FORT }}=785 \mathrm{~nm}$ for trapping rubidium [43]. The group of Chapman at Georgia Tech has developed a novel scheme whereby an optical dipole lattice transverse to the cavity axis acts as a 'conveyor belt' [51], transporting rubidium atoms into and out of a region of interaction with a weak probe field which is used to monitor the state of the system in real-time [42]. Beyond the use of optical traps, the groups of Walther and Blatt have made tremendous progress towards coupling single, trapped atomic ions to high-finesse cavities $[52,53]$. While these experiments have yet to enter the regime of strong coupling, practically limitless trap lifetimes and the powerful tools for coherent control which have already been developed within the ion trap community [54-57] hold great promise for future integration with optical cavities.

\section{Experiments with trapped atoms}

The development of a state-insensitive trap for strongly coupled atoms has not only extended the duration of individual transits far beyond the characteristic time scales for coherent atomfield coupling (i.e., $T \approx 3 \mathrm{~s}$ whereas $g_{0}^{-1} \approx 30 \mathrm{~ns}$ ), but also into a domain wherein complex experimental protocols can be performed using one-and-the-same atom. The cavity QED group at Caltech, working with atomic caesium, has begun exploring this domain with schemes for the generation of single photons, with measurements of the full vacuum-Rabi spectrum for just one atom, and with a new technique for in situ control of the system. 
(a)

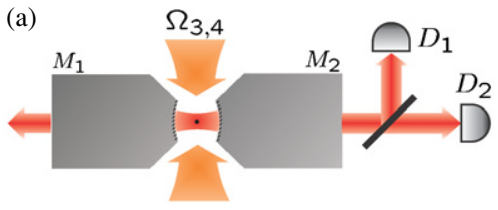

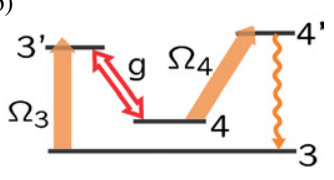

Figure 4. The one-atom laser. (a) Schematic diagram of the one-atom laser. Fields $\Omega_{3,4}$ are applied to a single caesium atom confined by an intracavity FORT, producing excitation in the TEM $\mathrm{TO}_{00}$ mode of the cavity. Photons which escape mirror $M_{2}$ are detected by single photon-counting avalanche photodiodes $D_{1,2}$. (b) Energy level diagram for the one-atom laser, as discussed in the text.

\subsection{The one-atom laser}

An optically driven single atom bound to a resonant cavity is, in some sense, a laser extrapolated to its fundamental, conceptual limit (theoretical descriptions of such devices have existed for a number of years [58-67]). In this analogy, the single atom serves as a gain medium which, as it 'lases', couples photons into the resonant mode of the cavity. However, the emission from such a device (like the device, itself) is qualitatively very unlike a conventional (semi-classical) laser, exhibiting a variety of manifestly quantum properties.

In a recent experiment to explore these phenomena [68, 69], a field $\Omega_{3}$ is applied from the side of the cavity (i.e., transverse to the cavity axis) which pumps the caesium atom from the $\left(6 S_{1 / 2}, F=3\right)$ ground state to the $\left(6 P_{3 / 2}, F^{\prime}=3^{\prime}\right)$ excited state (see figure 4$)$. The atomic population is rapidly transferred to $\left(6 S_{1 / 2}, F=4\right)$ via strong coupling to the cavity (which is tuned to be resonant with the $\left(6 P_{3 / 2}, F^{\prime}=3^{\prime} \rightarrow 6 S_{1 / 2}, F=4\right)$ transition), leaving a single excitation in the 'laser' mode. A second field $\Omega_{4}$, continuously applied to the atom, pumps to $\left(6 P_{3 / 2}, F^{\prime}=4^{\prime}\right)$ from which spontaneous emission returns the system to its (initial) ground state. As the resulting photons exit the cavity at a rate $2 \kappa$, their arrival times are recorded using a pair of single photon-counting avalanche photodiodes. Experimentally, we observe 'thresholdless lasing' and a maximum intracavity photon number which is rate-limited due to the (irreversible) recycling of the one atom through its energy levels by $\Omega_{3,4}$. Moreover, the one-atom laser exhibits photon antibunching and sub-Poissonian photon statistics, evidence that it is a manifestly quantum light source. For comparison with our experimental results, we have extensively analysed the theory of 'lasing' in the strong-coupling regime, as discussed in [69].

\subsection{Deterministic generation of single photons}

While the one-atom laser produces a nonclassical stream of photons, it is also possible to add an extra layer of control to the system in order to reliably and deterministically produce one photon on demand. We have shown that by iteratively pulsing the $\Omega_{3,4}$ beams, a single photon can be generated in the cavity mode with near unit efficiency [70]. Operationally, the $\Omega_{3}(t)$ pulse, in concert with an atom strongly coupled to a cavity on resonance with $\left(6 P_{3 / 2}, F^{\prime}=3^{\prime} \rightarrow 6 P_{1 / 2}, F=4\right)$, drives coherent, adiabatic transfer of atomic population between hyperfine ground states [71]. Simultaneously, this process introduces a single photon into the intracavity field, which subsequently escapes with a spatial profile fixed by the TEM $\mathrm{T}_{00}$ mode of the cavity and with a temporal profile determined by that of the $\Omega_{3}(t)$ pulse. A short pulse of $\Omega_{4}$ light incoherently repumps the atom to the $F=3$ manifold and resets the system to generate subsequent photons. 


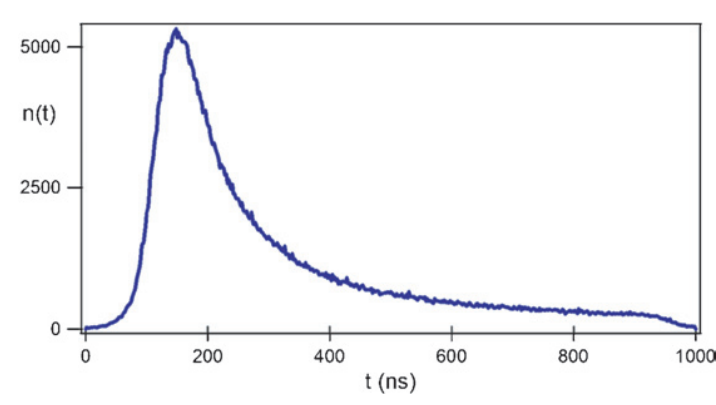

(a)

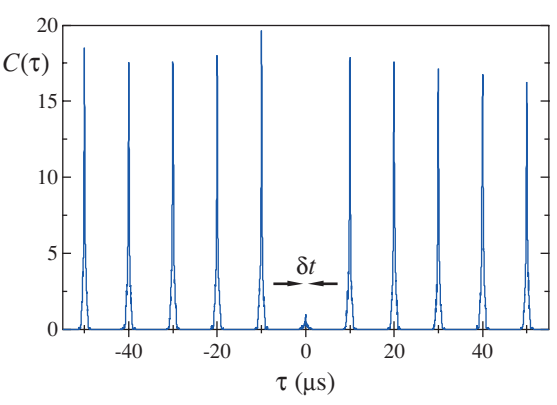

(b)

Figure 5. Single photon generation on demand. (a) The temporal profile of single photons generated from one caesium atom strongly coupled to a cavity [70]. The full-width at halfmaximum is $\tau_{\text {FWHM }}=120 \mathrm{~ns}$ and the long tail is the result of inhomogeneous preparation of initial Zeeman states. (b) The correlation function $C(\tau)$ as a function of time delay $\tau$ for counts from two single photon-counting photodiodes. The significant suppression of the peak at $\tau=0$ indicates that spurious two-photon events are rare.

In the regime of strong coupling, the rate $g(\mathbf{r})$ is sufficiently large that single photons are generated within the cavity with inferred efficiency $\phi_{G}=1.15 \pm 0.18$, where the uncertainty is determined from measurements of systematic losses and rare occurrences of two-photon events. Unpolarized photons escape the cavity with efficiency $(69 \pm 10) \%$ and are detected by two photoelectric detectors at the ports of a 50/50 beam splitter, leading to an overall detection efficiency of $\sim 2.4 \%$ (intracavity photon to photoelectric event). The temporal full-width at half-maximum of the photons is $\tau_{\mathrm{FHWM}}=120 \mathrm{~ns}$ for an $\Omega_{3}(t)$ pulse of uniform intensity over a duration of $1 \mu$ s (see figure 5(a)). The mean trap lifetime for a single atom during this process was $140 \mathrm{~ms}$, limited by heating from $\Omega_{3,4}$, allowing $1.4 \times 10^{4}$ single photons to be generated from each individual atom. The small probability of a two-photon event taking place is clearly evident from the suppression of a peak at $\tau=0$ in the correlation function between the two detectors, figure 5(b). As a metric for how closely this system approximates an ideal single-photon source we consider the quantity $R$, which quantifies the probability of two-photon events relative to a coherent state $(R \approx 1$ for a weak coherent state and approaches infinity for an ideal single-photon source). Averaged over all generation attempts, we measure $R=15.9 \pm 1.0$ which approaches $R \approx 150$ when considering only attempts occurring late in the trapping interval. We thereby demonstrate the predominantly singlephoton character of our source and hypothesize that rare two-photon events are principally the result of 'contamination' from rare occurrences in which two atoms are simultaneously loaded into the trap, both generating photons [70].

The non-negligible likelihood of two or more atoms being loaded into the FORT is an unavoidable consequence of our Poissonian technique for introducing cold atoms to the cavity. As in earlier work, a cloud of $\sim 10^{5}$ sub-Doppler cooled caesium atoms is released from a MOT and allowed to fall freely through the narrow space between the two cavity mirrors which, due to its prohibitive geometry, admits only a few atoms per attempt. We can control the mean number by adjusting the efficiency of the cooling beams which prepare the MOT, but not without detriment to the frequency with which we load single atoms. As a solution to this problem, the Caltech group has developed a technique whereby we can observe in real time discrete, 'step-like' increases in the transmission of probe beam as, one-by-one, multiple atoms are heated out of the FORT [72]. In future work, this protocol will provide an efficient means for ensuring that precisely $N=1$ atom is always loaded, or that a certain number 
$N>1$ is always loaded as required by some schemes for processing quantum information $[27,73]$.

\subsection{Intracavity Raman transitions and sideband cooling}

Beyond these advances enabled by the state-insensitive FORT [41, 70, 72], there exist a number of experimental parameters which remain inaccessible without more precise control over the atom and its motion. Among these are the location of the atom $\mathbf{r}$ relative to the QED field (and, implicitly, the range of coupling rates $g(\mathbf{r})$ that atom experiences as it moves within the mode of the cavity) and the characteristics of the ambient magnetic field at $\mathbf{r}$. In order to resolve these concerns and gain some a priori knowledge about atoms trapped in our FORT, we have developed a novel technique for driving stimulated Raman transitions between the hyperfine ground states of an atom in the FORT.

The presence of the cavity places certain geometric constraints on the beams which drive these transitions, so we have chosen to use the intracavity FORT field not only as a trap but also as one arm of the characteristic Raman $\Lambda$ configuration. The second arm is an auxiliary 'Raman' laser, phase-locked and orthogonally polarized relative to the FORT and offset by a frequency $\Delta_{\text {Raman }}=\Delta_{\mathrm{HF}}+\delta$ where $\Delta_{\mathrm{HF}}=9.192632 \mathrm{GHz}$, the caesium $6 S_{1 / 2}(F=3, F=4)$ splitting. $\delta$ is a variable frequency detuning. Both lasers drive the same cavity mode, with the Raman beam off cavity resonance by $\Delta_{\text {Raman }}$, and generate intracavity fields with individual Rabi frequencies $\left(\Omega_{\mathrm{FORT}}, \Omega_{\mathrm{Raman}}\right)$. In the limit that $\Delta^{\prime}=\left(v_{A}-v_{\mathrm{FORT}}\right) \gg\left\{\Omega_{F}, \Omega_{R}, \Delta_{\text {Raman }}, \delta, \gamma\right\}$, (where $v_{A}, v_{\mathrm{FORT}}$ are the frequencies of the caesium D2 line and the FORT laser, respectively) the net effective Rabi frequency is given by

$$
\Omega_{E}(t)=\frac{\Omega_{\mathrm{FORT}} \Omega_{\mathrm{Raman}}}{2 \Delta^{\prime}} .
$$

As the detuning $\delta$ is varied, it is possible to map out a spectrum of resonances between the various Zeeman and motional substates of the two hyperfine ground manifolds. After an atom is cooled into the FORT, a small magnetic field is applied along the cavity axis to break any degeneracy of Zeeman states so the atoms may be optically pumped into the $\left(F=3, m_{F}=0\right)$ ground state. The Raman laser at a fixed detuning $\delta$ is then pulsed ON for a duration $\tau_{\text {Raman }}$ in order to drive a $\theta_{\text {Raman }}=\tau_{\text {Raman }} \Omega_{E}$ rotation in the $(F=3,4)$ basis. Next, a probe pulse resonant with the $\left(F=4 \rightarrow F^{\prime}=5^{\prime}\right)$ transition detects whether the atom is in the $F=4$ ground state (using the type of cavity QED interaction discussed in the first section; large (small) transmission of the probe means the atom is decoupled from (coupled to) the cavity, namely in the $F=3(F=4)$ ground state). Many iterations of this procedure yield the probability $P(\delta)$ to drive a Raman transition at that specific detuning. As $\delta$ is varied, the result is a spectrum such as that in figure $6(\mathrm{a})$, showing a clearly resolved peak corresponding to the carrier $\left(F=3, m_{F}=0\right) \leftrightarrow\left(F=4, m_{F}=0\right)$ transition (with linewidth $\left.\Omega_{E}\right)$ as well as sidebands corresponding to $\Delta n= \pm 2$ vibrational transitions, which will be discussed in detail, below. By scanning over a broader range, it is possible to map out the full spectrum of $\Delta m_{F}=0$ transitions (or $\Delta m_{F}= \pm 1$, in a transverse bias field) from which information about the magnitude and direction of the local magnetic field can be extracted. Similarly, when the Raman pulse duration $\tau_{\text {Raman }}$ at $\delta \approx 0 \mathrm{MHz}$ is scanned in steps much smaller than $\Omega_{E}^{-1}$, it is possible to map out the so-called 'Rabi flopping,' the coherent transfer of atomic population between ground states (figure 6(b)).

Within the context of atomic systems bound to harmonic potentials, Raman transitions between quantized vibrational levels have become a standard tool. Raman sideband cooling is an essential component of experiments involving trapped ions [54] and has also been demonstrated for alkali atoms in free space [74, 75]. In the Fock basis we denote the 

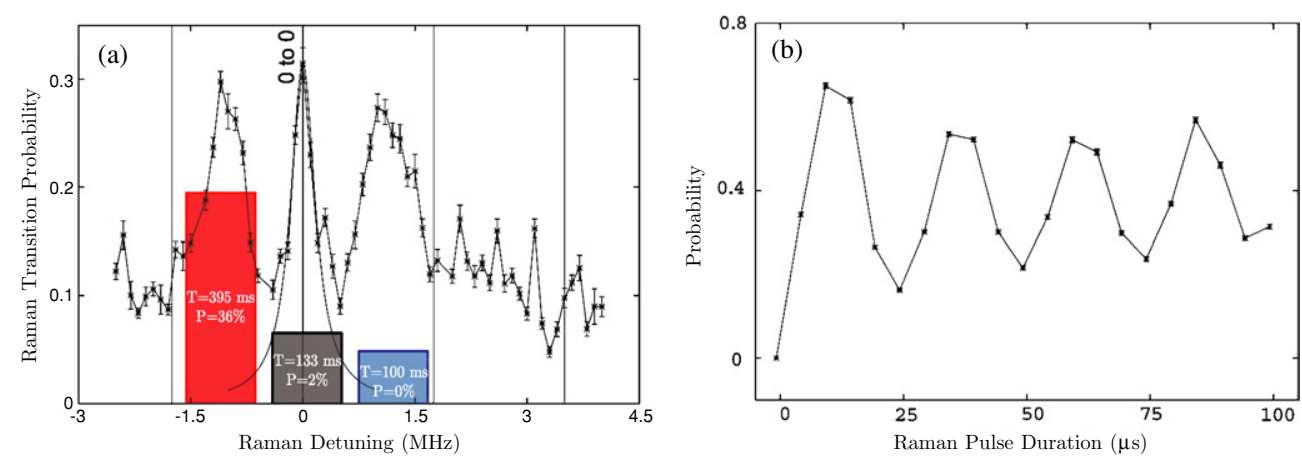

Figure 6. A Raman spectrum. (a) The 'carrier' transition $\left(m_{F}=0 \leftrightarrow m_{F}=0\right)$ and $\Delta n=2$ motional sidebands measured by driving stimulated Raman transitions between the hyperfine ground states of a single caesium atom confined in an intracavity FORT. Imposed over the trace are the results of cooling measurements undertaken on each of the resonances. The extended trap lifetime observed by driving the red transition constitutes empirical evidence of Raman sideband cooling. (b) 'Rabi flopping' on the $\Delta m_{F}=0$ 'carrier' transition as discussed in the text.

vibrational state of an atom in our FORT as $n$. By tuning our Raman beam (blue, red) of the carrier transition by twice the harmonic frequency of our FORT, it is possible to excite the atom from $n$ to $(n+2, n-2)$, the two sidebands observed in figure $6(\mathrm{a})$. Note that because the fields associated with the Raman and FORT beams are symmetric with respect to the boundary conditions set by the cavity, only even numbered $\Delta n$ transitions are allowed. The positions of the sidebands in the figure correspond reasonably well to the expectation $\pm 2 v_{0} \simeq \pm 1.1 \mathrm{MHz}$, where $v_{0}$ is the vibrational frequency for harmonic motion at the antinode of the FORT, which is independently determined from the FORT and caesium parameters. However, we do not yet understand the observed lineshapes nor the loss of contrast evidenced in figure 6, which may be due to the considerable anharmonicity of the FORT for weakly bound atoms. Preliminary attempts at cooling centre-of-mass motion by driving Raman vibrational sidebands empirically suggest that we can extend the lifetime of trapped atoms within our cavity, as indicated in the bar plots superimposed over the Raman scan. In a series of trials, atoms loaded into the FORT were driven on either of the blue, red or carrier transitions while being probed at regular intervals. For each, the corresponding trapping durations $T$, represented by the relative sizes of the bars, and probabilities $P$ that an atom loaded at the beginning of a $1 \mathrm{~s}$ long trial would survive until the end of the trial were recorded. On average, atoms driven on the red (cooling) sideband remained in the cavity substantially longer than those driven on the other motional transitions.

\subsection{The vacuum-Rabi spectrum of one-and-the-same atom}

Assisted by the capability to perform axial cooling using Raman transitions, our group was recently able to undertake measurements of the vacuum-Rabi spectrum for one-and-the-same atom. This is in contrast to the measurements discussed above [32, 37] and other recent observations made with atoms trapped in a FORT [76], all of which require averaging over a large ensemble of atoms (e.g., $10^{3}-10^{4}$ atoms in [76]). After a single atom is loaded into the FORT, a probe beam of frequency $\omega_{p}$, varied over a range near the atom-cavity resonance $\left(\omega_{A}=\omega_{C_{1}}\right)$, is mode-matched into the cavity, and the transmission of the probe $T\left(\omega_{p}\right)$ is recorded. After each probe interval, a cycle of Raman sideband cooling is interspersed before the probe frequency is iterated forwards and the process repeated. By choosing only those 


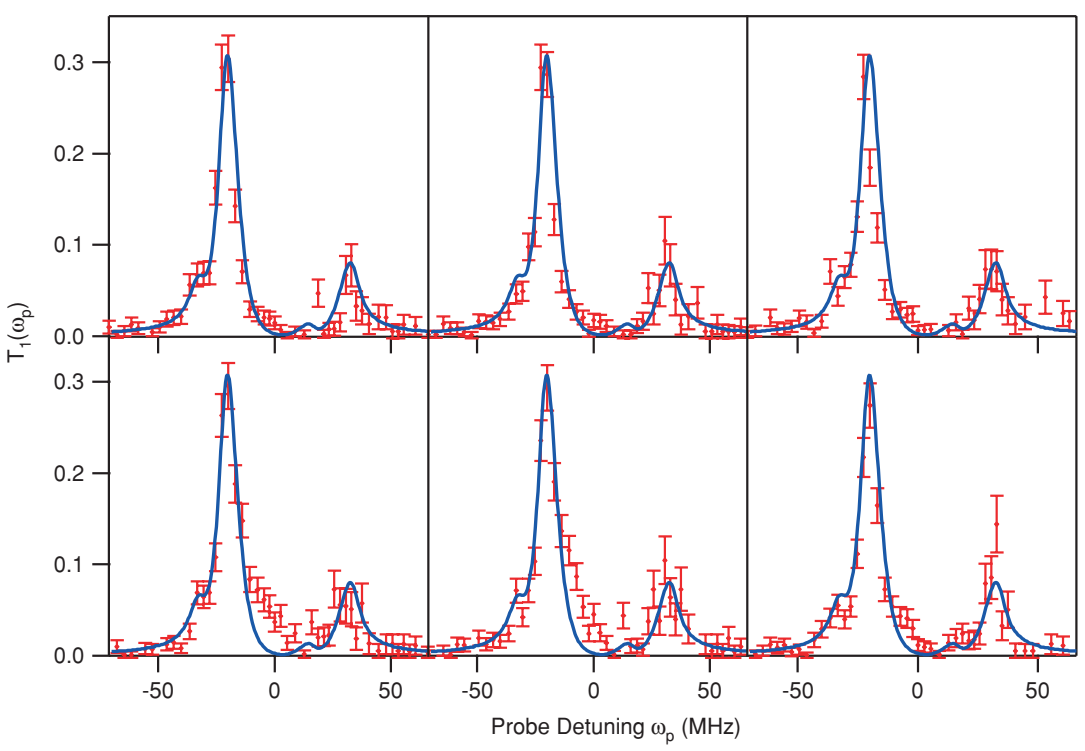

Figure 7. Vacuum-Rabi splitting of one-and-the-same atom. The complete vacuum-Rabi transmission spectra, $T\left(\omega_{p}\right)$ for six atoms, selected at random from a pool of 28 such spectra. The error bars represent statistical uncertainties. The blue trace is the solution of the master equation for the system [77].

trials for which the empty cavity resonance at $\omega_{p}=\omega_{C_{1}}$ is suppressed (i.e., those with strong resonant absorption indicating that an atom is coupled to the cavity), we are able to obtain the vacuum-Rabi spectrum for precisely one atom (six of which, for six separate atoms, were randomly selected and are shown in figure 7) [77].

For comparison, also included in figure 7 is the solution to the steady-state master equation for this system, incorporating only known experimental parameters and averaged over the top $1 / 3$ of FORT wells for which $g(\mathbf{r})$ is closest to its maximum value, $g_{0}$ (i.e., $g(\mathbf{r}) \gtrsim 0.87 g_{0}$ ). The results are in quite good agreement with the data, and the characteristic two peaked vacuum-Rabi structure is clearly present for each atom. The asymmetric features of the spectrum (i.e., peak heights, centroid locations) are principally the result of the small Zeeman state-dependent ac-Stark shifts induced by the FORT in conjunction with optical pumping effects due to the probe. In summation, these spectra contain detailed quantitative information about $g(\mathbf{r})$, indicating that atoms trapped and cooled within the FORT exist in a narrow range of near maximal values. This result is emblematic of the type of measurement which we expect the Raman technique to enable in the future.

\section{Conclusion}

We have discussed the evolution of experiments in optical cavity quantum electrodynamics, emphasizing those recent experiments enabled by intracavity state-insensitive optical dipole trapping. This work includes a demonstration of a one-atom laser, deterministic generation of single photons, the capacity for driving stimulated Raman transitions between hyperfine ground states of a trapped atom, and the observation of the vacuum-Rabi spectrum, the hallmark of strong coupling, for one-and-the-same atom. 
In recent years, many advances in cavity QED have been driven by an interplay with quantum information science [78]. In the near future, the tools discussed above will begin to play an important role as it becomes possible to perform complex quantum information protocols [27-30]. In particular it may even be possible, via Raman sideband cooling, to enter a regime requiring a quantized treatment for all degrees of freedom in QED, namely the state of the atom, its centre-of-mass motion, and the field to which it is coupled. This hearkens back to Einstein and his introduction of the quantum hypothesis, which, one hundred years ago, set the stage for the remarkable experimental advances of today.

\section{Acknowledgments}

This work was supported by the National Science Foundation (NSF), by the Caltech MURI Center for Quantum Networks, by the Advanced Research and Development Activity (ARDA), and by the Office of Naval Research (ONR). RM acknowledges support from the Army Research Office (ARO) QuaCGR programme.

\section{References}

[1] Stachel J (ed) 1989 The Collected Papers of Albert Einstein (Princeton, NJ: Princeton University Press) p 135

[2] Planck M 1901 Ann. Phys., Lpz. 4561

[3] Einstein A 1905 Ann. Phys., Lpz. 17132

[4] Einstein A 1906 Ann. Phys., Lpz. 20199

[5] Einstein A 1907 Ann. Phys., Lpz. 22180

[6] Einstein A 1909 Phys. Z. 10185

[7] Einstein A 1909 Phys. Z. 10817

[8] ter Haar D 1967 The Old Quantum Theory (Oxford: Pergamon)

[9] Mandel L and Wolf E 1995 Optical Coherence and Quantum Optics (Cambridge: Cambridge University Press)

[10] Jaynes E T and Cummings F W 1963 Proc. IEEE 5189

[11] Meystre P 1982 Progress in Optics vol 30 ed E Wolf (Amsterdam: Elsevier) p 261

[12] Kimble H J 1998 Phys. Scr. T 76127

[13] Carmichael H J 1993 An Open Systems Approach to Quantum Optics (Lecture Notes in Physics vol 18) (Berlin: Springer)

[14] Vernooy D and Kimble H J 1997 Phys. Rev. A 551239

[15] Vernooy D, Ilchenko V S, Mabuchi H, Streed E and Kimble H J 1998 Opt. Lett. 23247

[16] Buck J R and Kimble H J 2003 Phys. Rev. A 67033806

[17] Chiorescu I, Bertet P, Semba K, Nakamura Y, Harmas C J P M and Mooij J E 2004 Nature 431159

[18] Wallraff A, Schuster D I, Blais A, Frunzio L, Huang R-S, Majer J, Kumar S, Girvin S M and Schoelkopf R J 2004 Nature 431162

[19] Raimond J M, Brune M and Haroche S 2001 Rev. Mod. Phys. 73565

[20] Walther H 1998 Proc. R. Soc. L. A 454431

[21] Berman P (ed) 1994 Cavity Quantum Electrodynamics (San Diego: Academic)

[22] Rempe G, Thompson R J, Kimble H J and Lalezari R 1992 Opt. Lett. 17363

[23] Vahala K 2003 Nature 4046950

[24] Spillane S M, Kippenberg T J, Vahala K H, Goh K W, Wilcut E and Kimble H J 2005 Phys. Rev. A 71013817

[25] Mabuchi H and Doherty A C 2002 Science 2981372

[26] Lev B, Srinivasan K, Barclay P, Painter O and Mabuchi H 2004 Nanotechnology 15 S556

[27] Pellizzari T, Gardiner S A, Cirac J I and Zoller P 1995 Phys. Rev. Lett. 753788

[28] Duan L-M and Kimble H J 2004 Phys. Rev. Lett. 92127902

[29] Cirac J I, Zoller P, Kimble H J and Mabuchi H 1997 Phys. Rev. Lett. 783221

[30] Briegel H J et al The Physics of Quantum Information ed D Bouwmeester, A Ekert and A Zeilinger (Berlin: Springer) p 192

[31] Rempe G, Thompson R J, Brecha R J, Lee W D and Kimble H J 1991 Phys. Rev. Lett. 671727

[32] Thompson R J, Rempe G and Kimble H J 1992 Phys. Rev. Lett. 681132

[33] Thompson R J, Turchette Q A, Carnal O and Kimble H J 1998 Phys. Rev. A 573084

[34] Childs J J, An K, Otteson M S, Dasari R R and Feld M S 1996 Phys. Rev. Lett. 772901 
[35] Brune M, Schmidt-Kaler F, Maali A, Dreyer J, Hagley E, Raimond J M and Haroche S 1996 Phys. Rev. Lett. 761800

[36] Mabuchi H, Turchette Q A, Chapman M S and Kimble H J 1996 Opt. Lett. 211393

[37] Hood C J, Lynn T, Chapman M and Kimble H J 1998 Phys. Rev. Lett. 804157

[38] Hood C J, Lynn T W, Doherty A C, Parkins A S and Kimble H J 2000 Science 2871457

[39] Pinkse P W H, Fischer T, Maunz P and Rempe G 2000 Nature 404365

[40] Doherty A C, Lynn T W, Hood C J and Kimble H J 2001 Phys. Rev. A 63013401

[41] McKeever J, Buck J R, Boozer A D, Kuzmich A, Nägerl H-C, Stamper-Kurn D M and Kimble H J 2003 Phys. Rev. Lett. 90133602

[42] Sauer J A, Fortier K M, Chang M S, Hamley C D and Chapman M S 2004 Phys. Rev. A 69051804

[43] Maunz P, Puppe T, Schuster I, Syassen N, Pinkse P W H and Rempe G 2004 Nature 42850

[44] Ye J, Vernooy D W and Kimble H J 1999 Phys. Rev. Lett. 834987

[45] Metcalf H and van der Straten P 1999 Laser Cooling and Trapping (Berlin: Springer)

[46] Grimm R, Weidemüller M and Ovchinnikov Y B 2000 Adv. At. Mol. Opt. Phys. 4295

[47] Kimble H J 1999 ICOLS 99: Proc. 14 Int. Conf. on Laser Spectroscopy ed R Blatt, J Eschner, D Leibfried and F Schmidt-Kaler (Innsbruck: ICOLS)

[48] Hood C J 2000 Doctoral Dissertation California Institute of Technology

[49] Ido T, Isoya Y and Katori H 1999 J. Phys. Soc. Japan 682479

[50] McKeever J 2004 Doctoral Dissertation California Institute of Technology

[51] Kuhr S, Alt W, Schrader D, Müller M, Gomer V and Meschede D 2001 Science 293278

[52] Keller M, Lange B, Hayasaka K, Lange W and Walther H 2004 Nature 4311075

[53] Mundt A B, Kreuter A, Becher C, Leibfried D, Eschner J, Schmidt-Kaler F and Blatt R 2002 Phys. Rev. Lett. 89103001

[54] Monroe C, Meekhof D, King B, Jefferts S, Itano W, Wineland D and Gould P 1995 Phys. Rev. Lett. 754011

[55] Barrett M D et al 2004 Nature 429737

[56] Riebe $\mathrm{M}$ et al 2004 Nature $\mathbf{4 2 9} 734$

[57] Blinov B B, Moehring D L, Duan L-M and Monroe C 2004 Nature 428153

[58] Mu Y and Savage C 1992 Phys. Rev. A 465944

[59] Ginzel C, Briegel H-J, Martini U, Englert B-G and Schenzle A 1993 Phys. Rev. A 48732

[60] Pellizzarri T and Ritsch H 1994 Phys. Rev. Lett. 723973

[61] Horak P, Gheri K M and Ritsch H 1995 Phys. Rev. A 513257

[62] Meyer G M, Briegel H-J and Walther H 1997 Europhys. Lett. 37317

[63] Löffler M, Meyer G M and Walther H 1997 Phys. Rev. A 553923

[64] Meyer G M, Löffler M and Walther H 1997 Phys. Rev. A 56 R1099

[65] Meyer G M and Briegel H-J 1998 Phys. Rev. A 583210

[66] Jones B, Ghose S, Clemens J P, Rice P R and Pedrotti L M 1999 Phys. Rev. A 603267

[67] Kilin S Ya and Karlovich T B 2002 JETP 95805

[68] McKeever J, Boca A, Boozer A D, Buck J R and Kimble H J 2003 Nature 425268

[69] Boozer A D, Boca A, Buck J R, McKeever J and Kimble H J 2004 Phys. Rev. A 70023814

[70] McKeever J, Boca A, Boozer A D, Miller R, Buck J R, Kuzmich A and Kimble H J 2004 Science 3031992

[71] Parkins A S, Marte P, Zoller P and Kimble H J 1993 Phys. Rev. Lett. 713095

[72] McKeever J, Buck J R, Boozer A D and Kimble H J 2004 Phys. Rev. Lett. 93143601

[73] Duan L-M and Kimble H J 2003 Phys. Rev. Lett. 90253601

[74] Hamann S E, Haycock D L, Klose G, Pax P H, Deutsch I H and Jessen P S 1998 Phys. Rev. Lett. 804149

[75] Vuletić V, Chin C, Kerman A J and Chu S 1998 Phys. Rev. Lett. 815768

[76] Maunz P, Puppe T, Schuster I, Syassen N, Pinkse P W H and Rempe G 2004 Preprint quant-ph/045136

[77] Boca A, Miller R, Birnbaum K M, Boozer A D, McKeever J and Kimble H J 2004 Phys. Rev. Lett. 93233603

[78] Nielsen M A and Chuang I L 2000 Quantum Computation and Quantum Information (Cambridge: Cambridge University Press) 